\title{
Carbon nanotube-based mode-locked wavelength-switchable fiber laser via net gain cross section alteration
}

\begin{abstract}
We have proposed and demonstrated a carbon nanotube-based mode-locked erbium-doped fiber laser with switchable wavelength in the C-band wavelength region by varying the net gain cross section of erbium. The carbon nanotube is coated on a tapered fiber to form the saturable absorber for the purpose of mode-locking by exploiting the concept of evanescent field interaction on the tapered fiber with the carbon nanotube in a ring cavity configuration. The propagation loss is adjusted by inducing macrobend losses of the optical fiber in the cavity through a fiber spooling technique. Since the spooling radius can be gradually adjusted to achieve continuous tuning of attenuation, this passive tuning approach can be an alternative to optical tunable attenuator, with freedom of external device integration into the laser cavity. Based on this alteration, the net gain cross section of the laser system can be tailored to three different lasing wavelength ranges; 1533, 1560 rnlm and both (1533 and $1560 \mathrm{fllm}$ ) with the minimum pulse duration of $734 \mathrm{fs}$. The proposed design is simple and stable with high beam quality and good reliability for multiple applications.
\end{abstract}

Keyword: Carbon nanotube; Fiber laser; Mode-locking 